\title{
Identification of a Panax ginseng fruit fingerprint by HPLC-ESI-MS
}

\author{
H.F. Zhao' ${ }^{1}$, F.F. Xu' ${ }^{2}$, Y.T. Guo ${ }^{1}$ and H. Mi ${ }^{1}$ \\ ${ }^{1}$ Academy of Chinese Medical Sciences of Jilin Province, Changchun, China \\ ${ }^{2}$ Jilin Ji'an Yisheng Pharmaceutical Limited by Share Ltd., Ji'an, China \\ Corresponding author: $\mathrm{H}$. Mi \\ E-mail: hong_mi_au@163.com
}

Genet. Mol. Res. 15 (1): gmr.15017428

Received July 16, 2015

Accepted October 30, 2015

Published March 11, 2016

DOI http://dx.doi.org/10.4238/gmr.15017235

\begin{abstract}
Over many years, parts of Panax ginseng (root and rhizome) have been identified and applied for medical purposes as traditional Chinese herbal medicine. Recently, research has indicated that ginseng fruit also contains similar compounds and is as rich as the other parts of the ginseng. This discovery may dramatically improve the efficient of outputs derived from ginseng products. Here, a new technique combining high-performance liquid chromatography (HPLC) with electrospray ionization tandem mass spectrometry (ESI-MS) was employed to identify the fingerprint of $P$. ginseng fruit. Using HPLC, compounds that are important for medical purposes were extracted and purified. Combined with ESI-MS, the characteristic peaks (nine common peaks) of those compounds were identified, and the accuracy was confirmed by analysis using the Chromatographic Fingerprint Similarity Evaluation System (2004A edition). Overall, 15 batches of ginseng fruit had a similarity of more than $0.80,13$ batches of samples had a similarity between 0.97 and 0.99 , and two batches had a similarity less than 0.90 . The test solution and mobile phase selection was discussed. The HPLC-ESI-MS method can produce repeatable and reliable results and can be applied in the quality control of $P$. ginseng fruit.
\end{abstract}

Key words: Panax ginseng fruit; Electrospray ionization mass spectrometry; Fingerprint spectrum; High-performance liquid chromatography 


\section{INTRODUCTION}

Ginseng fruit is a dried ripe fruit belonging to the Araliaceae plant (Panax ginseng C.A. Mey). During the long history of Chinese medicinal herbs, the roots and rhizomes from ginseng have been used specifically for medical purposes. Both of these parts are very precious and named as one of the "Northeast Sambo", the three most valuable traditional treasures owned by people living in north east China. However, ginseng fruit has not traditionally been used for medical purposes. With the development of modern technology, researchers have explored ginseng in more depth, and have discovered that its fruit possesses similar components and pharmacological effects to other parts of ginseng (Zhao, 1979; Chen et al., 1986; Bai et al., 1987; Attele et al., 2002; Dey et al., 2002; Yu, 2002; Xie et al., 2002). Li and Teng (1978) analyzed ginsenosides and aglycone in different parts of ginseng and found that the saponin content in the fruit and flower was about 4-fold higher than in ginseng taproot; roots, flowers, and leaves, and about 2-fold higher in ginseng leaf. Fruits were found to contain panaxadiol, panaxatriol, and oleanolic acid. Yahara and Tanaka (1979) further studied the net content of ginsenodides in different parts of the ginseng monomer. These results implied that ginseng roots, stems, leaves, flowers, and fruits contain the ginsenosides Rb1, $\mathrm{Rb} 2, \mathrm{Rc}, \mathrm{Rd}, \mathrm{Re}$, and Rg1. Out of these parts, ginseng taproot contained the highest level of ginsenoside Rb1, ginseng stems and leaves contained the highest level of ginsenoside Rb2, and flowers and fruits contained the highest level of ginsenoside Re. This shows that ginseng fruits are of critical medicinal value and are as important as the other parts of ginseng. However, essential research into ginseng fruits has not drawn enough attention, especially compared to the medical application of ginseng and ginseng leaf, which have been included in the "People's Republic of China Pharmacopoeia" as national standards (Pharmacopoeia of the People's Republic of China, 2010). This greatly limits the application of ginseng fruits.

In addition, from an experimental point of view, the separation and identification of components of ginseng fruits is a big challenge to the researchers. Ginsenosides are the main chemical constituents of ginseng fruit. Usually, many steps, such as extraction, separation, and purification, have to be performed in order to obtain the final chemical components that are suitable for use in further measurements. Then, further approaches have to be applied to identify the separated components from the purified sample.

A common approach used to separate and analyze ginsenosides is high-performance liquid chromatography (HPLC). In general, the HPLC method is effective, rapid, sensitive, and reproducible, and has a wide range of applications; therefore, it has become the preferred method for use in chromatographic research (Harkey et al., 2001; Fuzzati, 2004). In this study, an HPLC fingerprint analysis method was established for ginseng fruits and combined with the liquid chromatography-mass spectrometry (LC-MS) method to identify the nine main components in ginseng fruits that have important medical properties. Fingerprinting is an analytical method used to characterize the multi-component features of traditional Chinese medicine, which can reflect the common components of distinct ingredients (Xie et al., 2006). This method is extensively used in the quality control process due to the high efficiency and has become an internationally recognized approach for its accuracy and reliability.

To enable further identification of ginsenosides in ginseng fruits, HPLC combined with electrospray ionization tandem mass spectrometry (HPLC-ESI-MS) technology was applied to effectively separate and analyze the ginsenosides and to determine their chemical structures according to their relative molecular weight and structural information based on mass spectrometry. ESI-MS is an advanced soft ionization mass spectrometry method. With ionization under mild 
conditions, the spectrum is simple but has high sensitivity (Fuzzati, 2004; Li et al., 2006; Han et al., 2007). The resulting peaks shown on the spectrum correspond to individual excimer ion contained in the testing compound and can directly reflect the relative molecular mass information of those ions. In addition, the simple sample preparation and short analysis time are also beneficial to the rapid and sensitive analysis of the main components of traditional Chinese medicine.

In total, 15 batches of samples were explored in this study. Twelve batches of ginseng fruit were collected from the major product base, Jilin Province, and three batches were obtained from a Chinese herbal medicine market. HPLC-ESI-MS was applied to identify a ginseng fruit fingerprint with characteristic peak structures, and to confirm this with high resolution in order to provide more comprehensive and accurate information. This method is better suited to the quality control and evaluation of ginseng fruit herbs. Following a standard procedure as mentioned above, a thorough analysis and evaluation led to the development of a theory used to assess the quality of ginseng fruits. Furthermore, the above information provides practical significance for the modernization and standardization of traditional Chinese medicine and the development of international standards. In addition, this research is also critical for the further exploitation of the medicinal value of ginseng fruits, expanding the range of medicinal ginseng, and to avoid wasting resources that could benefit the economy and society.

\section{MATERIAL AND METHODS}

\section{Sample preparation and instrumentation}

\section{Sample collection}

In total, 15 batches of ginseng fruit products were provided by Jilin Yisheng Pharmaceutical Co., Ltd., Ji'an, Jilin, China. Among them, three batches were purchased from the Yasukuni Medicine Market, Anguo, Hebei, China, and twelve batches, were purchased as follows: two batches were from Jingyu, Jilin; two batches were from Fusong, Jilin; three batches were from Ji'an, Jilin; two batches were from Changbai, Jilin; two batches were from Linjiang, Jilin; one batch was from Huinan, Jilin; and three batches were from Anguo, Hebei. All the products were identified as the fruit of Panax ginseng Meyer by Professor Xu Guojing, from Jilin Academy of Traditional Chinese Medicine Center: ginsenoside Re (110754-201123), ginsenoside Rg1 (110703-201027), ginsenoside Rf (111719-200703), ginsenoside Rb1 (110704201122), ginsenoside Rg2 (111779-200801), ginsenoside Rc (111686-201,002), ginsenoside Rb2 (111715-200802), ginsenoside Rb3 (111686-201002), and ginsenoside Rd (111818-201001). The reference standard was purchased from the National Institutes for Food and Drug Control (NIFDC), Beijing, China.

\section{Solution preparation}

\section{Preparation of the reference solution}

Ginsenoside $\mathrm{Re}$ is the main component of ginseng fruit, and is present at the highest level of all saponin components. Therefore, ginsenoside $\mathrm{Re}$ was chosen as a reference. Ginsenoside $\operatorname{Re}(92.7 \%)$ from the NIFDC was accurately weighed, dissolved in methanol, and placed in $1 \mathrm{mg} /$ $\mathrm{mL}$ aliquot solution to form the reference solution for further use. 


\section{Preparation of the test solution}

About $2 \mathrm{~g}$ ginseng fruit powder was accurately weighed out and added to $100 \mathrm{~mL}$ water. The solution was boiled twice for $\sim 1 \mathrm{~h}$ each. The filtered solution was combined and extracted twice by using $80 \mathrm{~mL}$ chloroform per extraction. The chloroform layer was subsequently discarded. Water-saturated butanol was added to extract the solution five times. The combined butanol layers were evaporated and dried. The residue was dissolved in methanol and diluted to $10 \mathrm{~mL}$ in a volumetric flask. After shaking, a $0.45-\mu \mathrm{m}$ microporous membrane was used to filter out the residual and the filtrate was kept for use as the test solution.

\section{Instrumentation (HPLC, LC-MS)}

LC-10AT HPLC (Shimadzu Scientific Inst., Kyoto, Japan) was applied to separate and analyze the samples. The separation column was a TC-C18 (250 x $4.6 \mathrm{~mm}, 5 \mu \mathrm{m}$; Agilent Technologies, Santa Clara, CA, USA). The column temperature was maintained at $35^{\circ} \mathrm{C}$ and the flow rate was set as $1.0 \mathrm{~mL} / \mathrm{min}$. The detection wavelength was set to $203 \mathrm{~nm}$. The injected sample volume was $20 \mu \mathrm{L}$. Mobile phase A was $100 \%$ acetonitrile and phase B was $0.05 \%$ phosphoric acid. The conditions for gradient elution were as follows: 0-12 $\min (19 \% \mathrm{~A}), 12-34 \mathrm{~min}(19-24 \% \mathrm{~A}), 34-60$ $\min (24-33 \% \mathrm{~A})$, and $60-95 \mathrm{~min}(33-35 \% \mathrm{~A})$, with an initial balance time of $20 \mathrm{~min}$. The theoretical plate number was calculated based on the ginsenoside Re (\#2 peak) and was not less than 5000. HPLC-grade acetonitrile and methanol were purchased from Fisher Scientific, Ptttsburgh, PA, USA. All other reagents were analytical grade and purchased from Beijing Chemical Works, Beijing, China, and distilled water was used. An LCQ ${ }^{\mathrm{TM}}$ ion trap benchtop mass spectrometer with an electrospray ionization source (Thermo Finnigan LLC, San Jose, CA, USA) was used as an ion trap detector. The scanning range was 200-1300 $U$ with the negative ion mode. The spray voltage was $5.0 \mathrm{kV}$ and the temperature of the metal capillary was kept at $280^{\circ} \mathrm{C}$. A Cschrom Plus (Scientific System, Inc., State College, PA, USA) chromatography workstation was also used.

\section{RESULTS}

\section{HPLC methodological study}

\section{Precision test}

Six sets of ginseng fruit test solutions from the same batch were continuously injected into the HPLC and each process followed the same procedure. The relative standard deviations (RSD) of the total peak relative retention time and the peak area ratio were $0.18-0.85$ and $1.01-1.94 \%$, respectively. Those results indicated that the fingerprint testing requirements for this HPLC setup were satisfied.

\section{Stability test}

Ginseng fruit test solution from the same batch was removed to conduct the HPLC stability test. An aliquot of the test solution was removed at $0,4,8,12$, and $24 \mathrm{~h}$. The resolved total peak RSD of the relative retention time for each sample was between 0.11 and $1.02 \%$ and the peak area ratio was between 0.15 and $1.65 \%$. These results provide solid evidence that the test solution was very stable over the $24-\mathrm{h}$ test period under the experimental temperature (room temperature). 


\section{Replication test}

Six sets of ginseng fruit test solutions were prepared from the same batch following the same procedure. The resolved total peak RSD of the relative retention time for each sample was between 0.15 and $0.82 \%$ and the peak area ratio was between 0.91 and $1.63 \%$. These results satisfied the test standard of the HPLC fingerprint.

\section{Ginseng fruit fingerprint established by HPLC}

Based on the above HPLC criteria, fingerprints of the 15 batches of ginseng fruit were measured and the ginsenoside Re peak (peak 2), which is the main component of the ginseng fruit, was set as the reference peak. The relative retention time of each peak was calculated. The RSD of the relative retention time of common peaks for all 15 batches was less than $1 \%$, which is in line with the requirements of fingerprints by the HPLC method. Applying the standard "Chromatographic Fingerprint Similarity Evaluation System 2004A edition", stated by the State Pharmacopoeia Commission to the fingerprints of 15 batches of samples, the resolved spectra are plotted in Figure 1. The application was set using sample 2 (peak 2) as the reference spectra, the "Width of the Time window is 0.1 min" was used, the median method was applied to the calculation, and nine characteristic peaks were selected for multi-point calibration and automatic matching. The matched sample spectra overlapped with the original spectra and the final results are plotted in Figure 2.

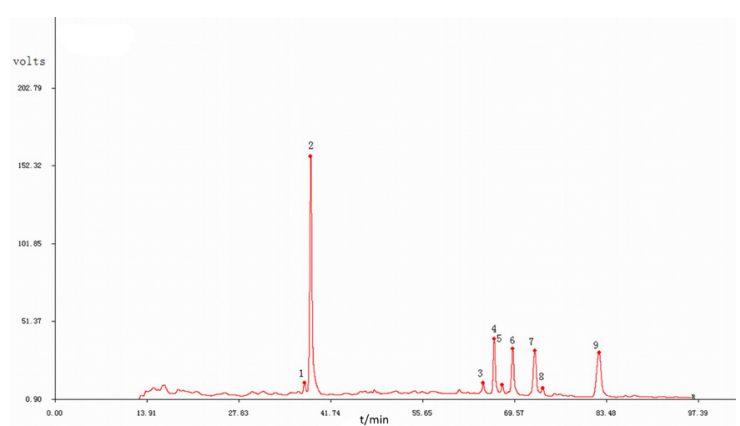

Figure 1. HPLC standard spectrum of fingerprint of Panax ginseng fruit. This standard spectrum was obtained under the standard from "Chromatographic Fingerprint Similarity Evaluation System 2004A edition", stated by the State Pharmacopoeia Commission to the fingerprints of $P$. ginseng fruit. Among the nine characteristic peaks, the number 2 peak is the highest and set up as the reference peak.

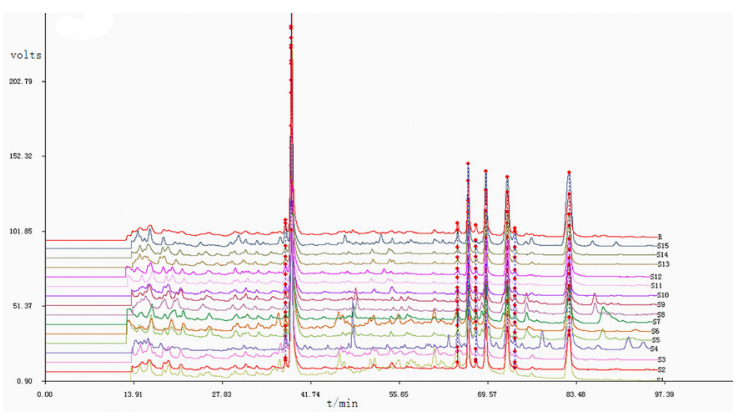

Figure 2. Overlaid HPLC spectrum from 15 habitats match the standard fingerprint of Panax ginseng fruitshown in Figure 1. 


\section{Identification of common peaks in the ginseng fruit fingerprint by MS}

Using the HPLC-ESI-MS technique, in-source collision-induced dissociation (CID) experiments by ESI-MS, the corresponding compound structural information of 1-9 common peaks was obtained. The experimental data are presented in Table 1. In-source CID experiments, [MH]- ion fragmentation occurred. These fragments can provide information about the type of ginsenoside, and about the type and connection sequence of glycosylation. Compounds 4, 6, 7, 8, and 9 (ginsenosides Rb1, Rc, Rb2, $\mathrm{Rb3}$, and $\mathrm{Rd}$ ) generated weak $\mathrm{m} / \mathrm{z} 459$ ions following loss of glycosylation. This indicated that these ginsenosides are of the panaxadiol type (Cui et al., 2000; Guo et al., 2003; Li et al., 2005; Shi et al., 2006; Zheng et al., 2012; Huang et al., 2013). [MH] ions from compounds 1, 2, 3, and 5 (ginsenosides Rg1, $\mathrm{Re}, \mathrm{Rf}$, and $\mathrm{Rg} 2$ ) generated sapogenin ions with $\mathrm{m} / \mathrm{z} 475$ ion. This indicated that they are panaxatrioltype saponins (Cui et al., 2000; Guo et al., 2003; Li et al., 2005; Shi et al., 2006; Zheng et al., 2012; Huang et al., 2013). [MH] ion cleavage of ginsenosides only occurred when the glycosidic bond was cleaved, and the charge was retained in the side of sapogenin. Therefore, depending on the type of debris, we can speculate the type of connection between the sugar chain and aglycone.

Table1.In-sourcecollision-induceddissociation(CID)dataofHPLC1-9peaksusingthenegativeionmode(m/zvalues).

\begin{tabular}{|c|c|c|c|c|}
\hline Peak & Components & $t_{R}(\min )$ & {$[\mathrm{M}-\mathrm{H}]^{-}(\mathrm{m} / \mathrm{z})$} & Mass spectra of in-source CID $(\mathrm{m} / \mathrm{z})$ \\
\hline 1 & $\mathrm{Rg} 1$ & 36.939 & 799 & 637[M-Glc-H]',475[M-2Glc-H] $]^{-}$ \\
\hline \multirow[t]{2}{*}{2} & $\operatorname{Re}$ & 37.901 & 945 & 799[M-Rha-H]',783[M-Glc-H]', \\
\hline & & & & 637[M-Glc-Rha-H]-,475[M-2Glc-Rha-H] ${ }^{-}$ \\
\hline 3 & $\mathrm{Rf}$ & 63.968 & 99 & $637[\mathrm{M}-\mathrm{Glc}-\mathrm{H}]^{-}, 475[\mathrm{M}-2 \mathrm{Glc}-\mathrm{H}]^{-}$ \\
\hline \multirow[t]{2}{*}{4} & $\mathrm{Rb} 1$ & 65.576 & 1107 & 945[M-Glc-H]',783[M-2Glc-H]; \\
\hline & & & & $621[\mathrm{M}-3 \mathrm{Glc}-\mathrm{H}]^{-}, 459[\mathrm{M}-4 \mathrm{Glc}-\mathrm{H}]^{-}$ \\
\hline 5 & $\mathrm{Rg} 2$ & 66.872 & 783 & $621[\mathrm{M}-\mathrm{Glc}-\mathrm{H}] ;, 475[\mathrm{M}-\mathrm{Glc}-\mathrm{Rha}-\mathrm{H}]^{-}$ \\
\hline \multirow[t]{2}{*}{6} & $\mathrm{Rc}$ & 68.124 & 1077 & 945[M-Ara(f)-H] $; 783[\mathrm{M}-\mathrm{Ara}(\mathrm{f})-\mathrm{Glc}-\mathrm{H}]^{-}$, \\
\hline & & & & $621[\mathrm{M}-\mathrm{Ara}(\mathrm{f})-2 \mathrm{Glc}-\mathrm{H}]^{-}, 459[\mathrm{M}-\mathrm{Ara}(\mathrm{f})-3 \mathrm{Glc}-\mathrm{H}]^{-}$ \\
\hline \multirow[t]{2}{*}{7} & $\mathrm{Rb} 2$ & 71.194 & 1077 & 945[M-Ara(p)-H];,783[M-Ara(p)-Glc-H] \\
\hline & & & & $621[M-A r a(p)-2 G I c-H] ; 459[M-A r a(p)-3 G l c-H]$ \\
\hline \multirow[t]{2}{*}{8} & $\mathrm{Rb} 3$ & 72.297 & 1077 & 915[M-Glc-H] ], 783[M-xyl-Glc-H]', \\
\hline & & & & $621[\mathrm{M}-\mathrm{xyl}-2 \mathrm{Glc}-\mathrm{H}]^{-}, 459[\mathrm{M}-\mathrm{xyl}-3 \mathrm{Glc}-\mathrm{H}]^{-}$ \\
\hline \multirow[t]{2}{*}{9} & $\mathrm{Rd}$ & 80.203 & 945 & 783[M-Glc-H]',621[M-2Glc-H] \\
\hline & & & & 459[M-3Glc-H] \\
\hline
\end{tabular}

For example, in the LC-MS spectrum, the first order spectrum of the quasi-molecular ion $[\mathrm{MH}]$ - for compound 2 was $\mathrm{m} / \mathrm{z} 945$ and in the in-source CID spectrum, m/z 799, 783, 637, and 475 and other fragment ions were observed. Among them, the $\mathrm{m} / \mathrm{z} 783$ fragment ion was generated by the $\mathrm{m} / \mathrm{z} 945$ ion through the loss of a hexose group, m/z 799 ion was a fragment ion generated by the $\mathrm{m} / \mathrm{z} 945$ ion through the loss of a deoxyhexose group, the $\mathrm{m} / \mathrm{z} 637$ ion was also generated as a fragment ion by an $\mathrm{m} / \mathrm{z} 945$ fragment ion simultaneously losing a hexose group and a deoxyhexose $\mathrm{s}$, and the $\mathrm{m} / \mathrm{z} 475$ ion is a fragment ion produced after $\mathrm{m} / \mathrm{z} 945$ loses all glycosylation. The generation of $\mathrm{m} / \mathrm{z} 475$ ions indicates that this ginsenoside is a panaxatriol-type saponin. Based on identical information of ginsenoside Re obtained by ESI-MS, and the consistent chromatographic retention time, this peak can be identified as the ginsenoside $\mathrm{Re}$.

Another example is provided for compounds 6 and 7 isomers ([MH]', m/z 1077). These two 
compounds have the same CID fragment ions ( $\mathrm{m} / \mathrm{z} 945,783,621,459)$. In the CID spectra, $\mathrm{m} / \mathrm{z}$ 1077 was observed to lose the first ion pentose group giving an $\mathrm{m} / \mathrm{z} 945$ ion, while $\mathrm{m} / \mathrm{z} 783$ ion was generated by losing a five-carbon sugar and a six-carbon glycosyl from m/z 1077, an m/z 621 ion was generated by the loss of a pentose ionic group from $\mathrm{m} / \mathrm{z} 1077$, and the subsequent loss of two hexose groups, and an $\mathrm{m} / \mathrm{z} 459$ ion was generated by the loss of all glycosylation from an $\mathrm{m} / \mathrm{z} 1077$ ion. The generation of an $\mathrm{m} / \mathrm{z} 459$ ion suggests that these two were panaxadiol-type ginsenoside saponins. Based on the above analysis, and on the comparison of the standard retention time of the compound spectrums, it can be concluded that these two ginsenosides were Rc and Rb2, respectively. Similarly, when compared and identified using other spectra, the CID data (in Table 1) and attribution (Figure 3) were identical to previous reports (Cui et al., 2000; Guo et al., 2003).

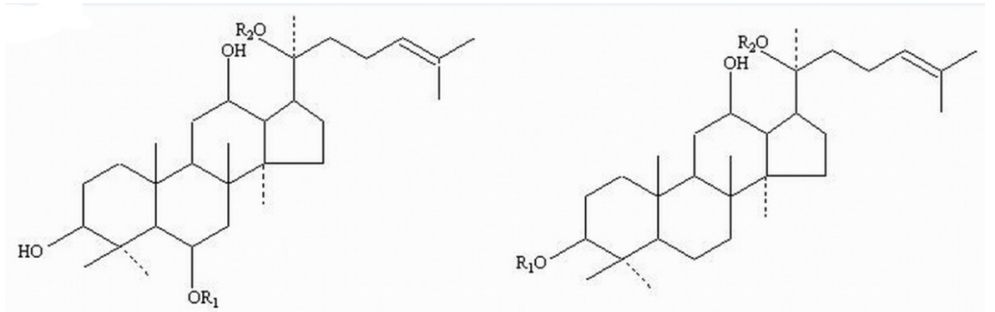

Figure 3. Structures of the nine ginsenosides in Panax ginseng fruit (the number is identical to the peak number in Figure 1). The radicals for each ginsenosides are listed as follows: $1 . \mathrm{R} 1=-\mathrm{glu}, \mathrm{R} 2=-\mathrm{glu} ; 2 . \mathrm{R} 1=-\mathrm{glu}(2-1) \mathrm{rha}, \mathrm{R} 2$ $=-g l u ; 3 . R 1=-g l u(2-1)$ glu, R2 = -H; $4 . \mathrm{R} 1=-$ glu(2-1)glu, R2 = -glu(6-1)glu; 5. R1 = -glu(2-1)rha, R2 = -H; $6 . \mathrm{R} 1=$ -glu(2-1)glu, R2 = -glu(6-1)araf; 7. R1 = -glu(2-1)glu, R2 = -glu(6-1)arap; 8. R1 = -glu(2-1)glu, R2 = -glu(6-1)xyl; 9. R1 $=-$ glu(2-1)glu, R2 = -glu.

\section{Evaluation of the similarity of 15 batches of ginseng fruit}

Following calculation using the Chromatographic Fingerprint Similarity Evaluation System (2004A), the similarity of results obtained from 15 batches of ginseng fruit is shown in Table 2 . The similarity of 13 batches was greater than 0.9 . Among the 13 batches, 12 were obtained mainly from cities inside Jilin Province and the remainder was a commercial sample obtained from a Chinese herbal medicine market. All 13 batches had a high degree of similarity, which indicates that the quality of ginseng in Jilin Province is very high. The similarity of the other two batches obtained from a Chinese herbal medicine market was below 0.90 .

Table 2. Similarity calculation of the HPLC fingerprint of 15 batches of Panax ginseng fruit.

\begin{tabular}{l|l|c}
\hline No. & Origin Collection date & Resemblance \\
\hline 1 & jian-1 2012-7 & 0.992 \\
\hline 2 & jian-2 2012-7 & 0.999 \\
\hline 3 & jian-3 2012-7 & 0.997 \\
\hline 4 & anguo-1 2012-1 & 0.855 \\
\hline 5 & anguo-2 2012-6 & 0.976 \\
\hline 6 & jingyu-1 2012-7 & 0.989 \\
\hline 7 & jingyu-2 2012-7 & 0.990 \\
\hline 8 & fusong-1 2012-7 & 0.992 \\
\hline 9 & fusong-2 2012-7 & 0.992 \\
\hline 10 & huinan 2012-7 & 0.999 \\
\hline 11 & changbai-1 2013-8 & 0.995 \\
\hline 12 & changbai-2 2013-8 & 0.999 \\
\hline 13 & linjiang-1 2013-8 & 0.999 \\
\hline 14 & linjiang-2 2013-8 & 0.999 \\
\hline
\end{tabular}




\section{DISCUSSION}

\section{Preparation of the test solution}

The test solution was prepared by two different methods, via water extraction and ethanol extraction. Apparently, resolution of the chromatographic peak of the samples obtained via ethanol extraction was low but with a high baseline. Therefore, the water extraction method was selected. The comparison is shown in Figure 4. During the purification process, macroporous resin adsorption and extraction was adopted to purify the ingredients from the extracted solution. The results obtained following macroporous resin adsorption showed that there was a large loss of active ingredients, and it was a complicated process with low reproducibility. The results are shown in Figure 5 . The extraction method was applied for the purification of the sample. After optimizing the duration of water extraction, boiling, and extraction, the optimal conditions were identified as follows: boiling water twice for $1 \mathrm{~h}$ each, use of water-saturated n-butanol to extract the test solution five times. Based on this protocol, the resolved chromatogram peaks were found to have a better resolution, peak shape, and baseline. This procedure was used to prepare ginseng fruit test solutions.
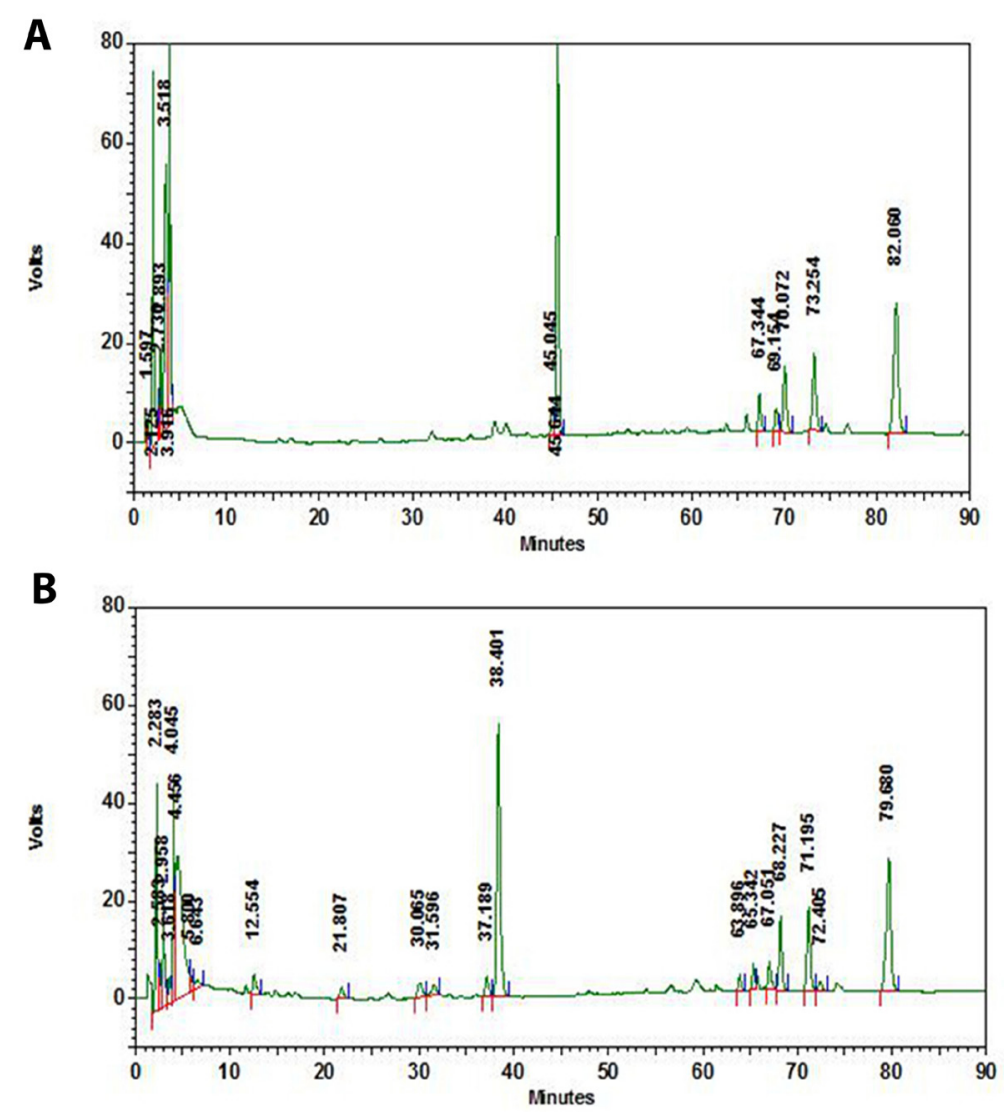

Figure 4. HPLC spectrum of test solution extracted by (A) ethanol extraction method or (B) water extraction method. The latter one showed the better resolution and low baseline from the spectrum. Therefore, water extraction method was used in preparing test solution. 

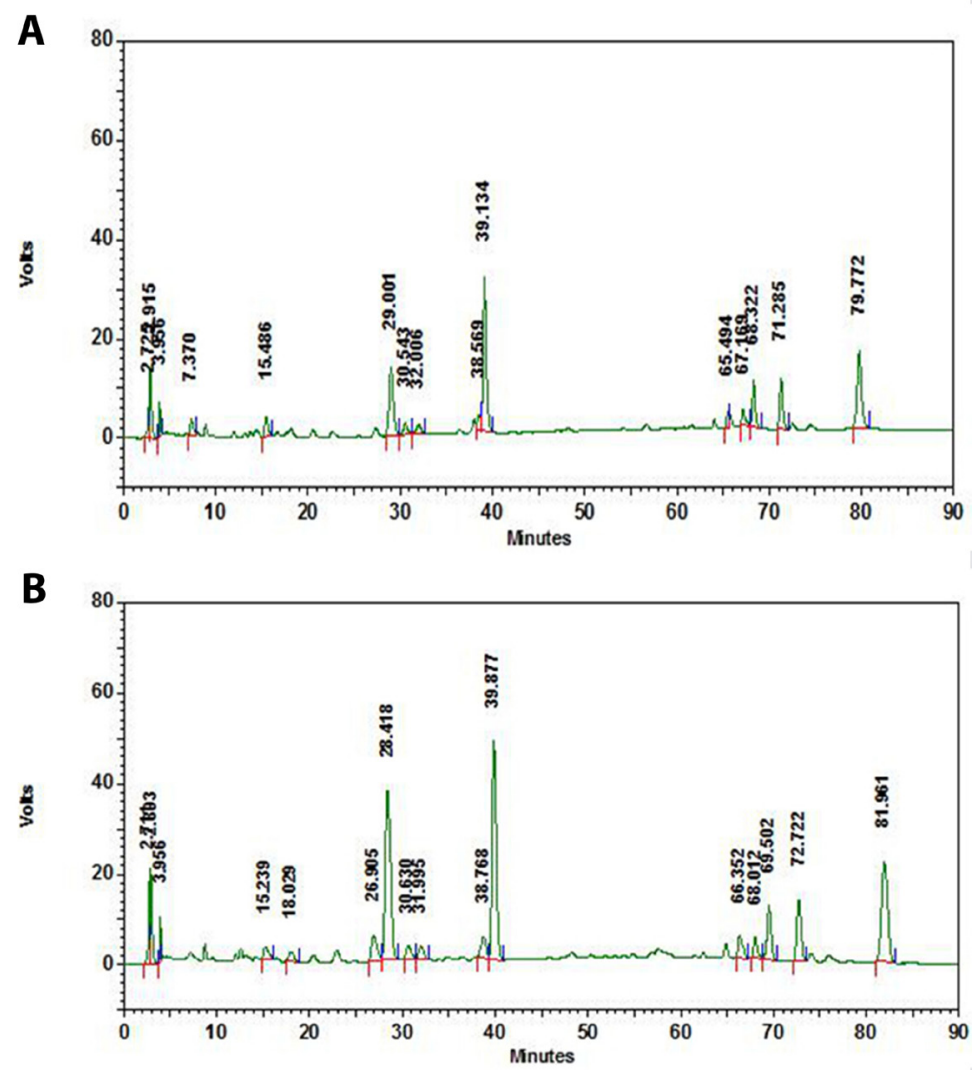

Figure 5. HPLC spectrum of purified test solution after applied by (A) macroporous resin adsorption method or (B) extraction method. Extraction method provided a simple process with high reproducibility and less sample lost. This method was applied to purify the test solution.

\section{Investigation of the mobile phase}

In this experiment, methanol-water, acetonitrile-water, and acetonitrile- $0.05 \%$ phosphoric acid solution were used as the mobile phase (Li et al., 2005; Shi et al., 2006; Huang et al., 2013). The result showed that methanol-water produced fewer and misshapen peaks in the chromatogram, and had lower separation; the acetonitrile-water system resulted in more peaks in the chromatogram and a clean baseline, but not good separation of individual peaks. Further improvement of the acetonitrile- $0.05 \%$ phosphoric acid system increased the number of chromatogram peaks, had a low baseline, and led to better resolution of each chromatographic peak. An acetonitrile- $0.05 \%$ phosphoric acid-based test solution was chosen as the optimal mobile phase. This comparison is shown is Figure 6.

In this paper, an HPLC-ESI-MS detection system was established to identify the fingerprint of ginseng fruit and to obtain structural information about the chemical component of saponin compounds in ginseng fruits. Saponins are the main medical components, and are usually polarized and less volatile with a high molecular weight. These characters have led to great difficulties in their separation, purification, and structural identification. In this paper, the structure 
of saponin compounds in ginseng fruits was characterized by anion tandem mass spectrometry, which indicated that multilevel saponin compound fragmentation is closely related to the structure of the compound. By analyzing anion ESI-MS first order spectra, molecular weight information of saponin compounds can be determined. Furthermore, by selecting the target ions through a multi-level series function, a multistage collision can be applied. In this way, information on saponin compounds may be determined as follows: 1) the type of sapogenin (diol or glycerol type); 2) the type and quantity of the attached glycosyl; and 3) the connection sequence of a sugar chain. Isomers with the same qualities can be distinguished by the characteristics of the cleaved ions. Experimental results showed that this method can quickly result in the identification of ginseng saponin compounds and provide structural information.
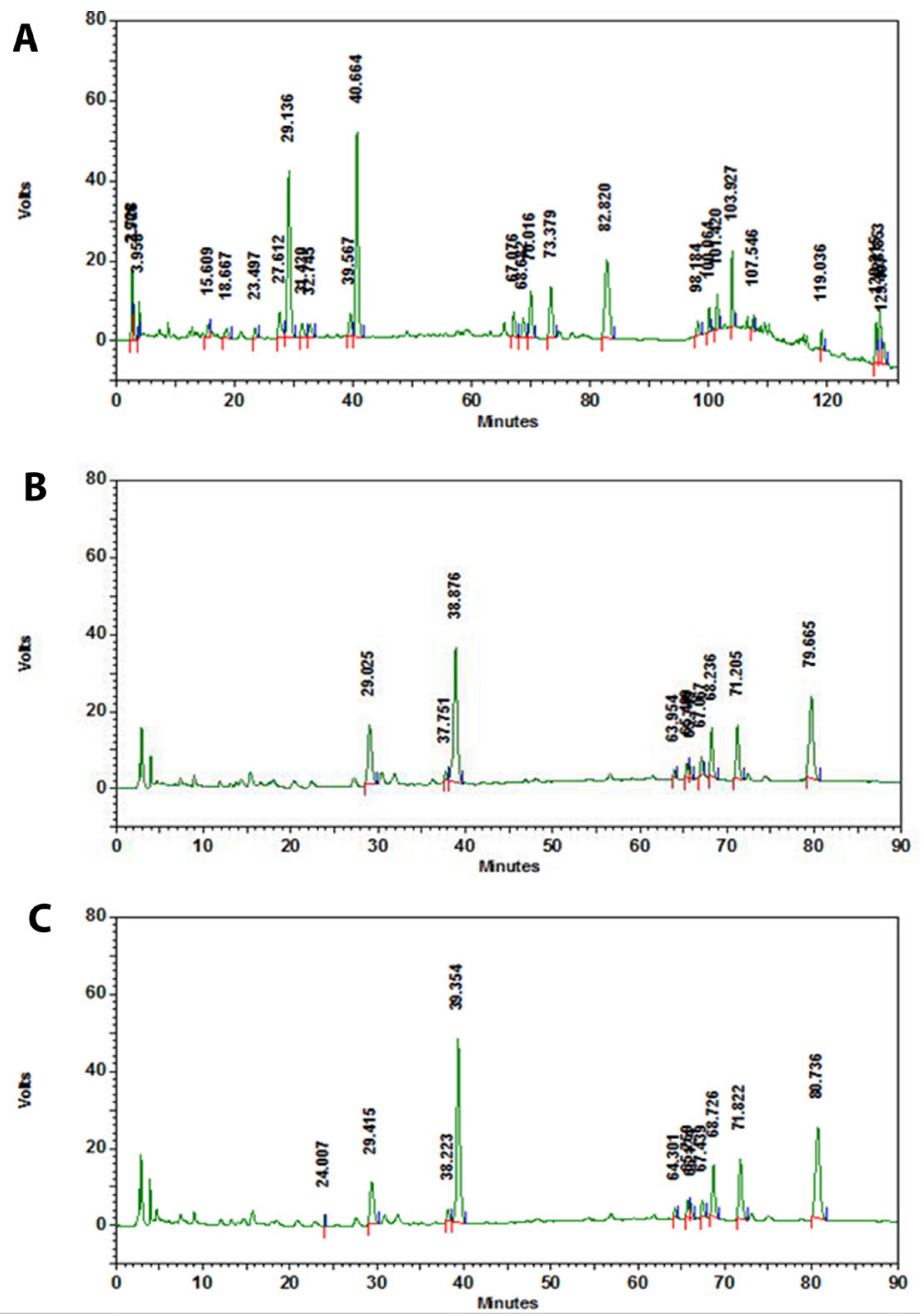

Figure 6. HPLC spectrum of three different solutions as the mobile phase. A. Methanol-water. B. Acetonitrile-water. C. Acetonitrile- $0.05 \%$ phosphoric acid solution. The acetonitrile- $0.05 \%$ phosphoric acid system offered the accurate chromatogram peak information and the best resolution for individual peak with relatively low baseline. This mobile phase was adopted in the experiment. 
Results obtained from the analysis of 15 different batches showed that although corresponding characteristic component peaks were presented in each batch, the peak areas differed. Among them, 12 batches were originally from Jilin Province and three batches were from a Chinese herbal medicine market. Results obtained through similarity evaluation software showed that the similarity of 13 batches is greater than 0.97 , which accounted for $86.7 \%$ of all samples. For the remaining two batches, it was less than 0.90 . These two batches are commercially available medicinal herbs. These data suggest that the quality of ginseng fruit was much higher when they were obtained from their main place of origin, Jilin Province.

The combined use of HPLC and MS is the best approach to identify fingerprints of characteristic peaks in order to confirm the structure of ginseng fruit. The HPLC-ESI-MS technique can be used to obtain a stronger characteristic fingerprint, which is more comprehensive, contains accurate information, and is better suited to the control and evaluation of ginseng fruit quality.

Currently, due to a lack of national standards in the harvesting market, the quality of commercially available ginseng fruits is inconsistent because of irregular harvesting, processing, and preservation processes at different locations. Establishing quality standards should be carried out immediately and the control and regulation of medicinal resources is very critical. Combined with modern approaches, the medical purpose of ginseng fruits can obtain the full support of the government. Therefore, more people could benefit from this development.

\section{Conflicts of interests}

The authors declare no conflict of interest.

\section{ACKNOWLEDGMENTS}

Research supported by a major project of the Scientific and Technological Achievements Transformation of Jilin Province (\#20115090) and the "2012" Postdoctoral research of Jilin Province.

\section{REFERENCES}

Attele AS, Zhou YP, Xie JT, Wu JA, et al. (2002). Antidiabetic effects of Panax ginseng berry extract and the identification of an effective component. Diabetes 51: 1851-1858. http://dx.doi.org/10.2337/diabetes.51.6.1851

Bai XG, Xu JD, Chen GR and Jiang XK (1987). Chemical study of ginseng fruit-structural identification of saponin GF-I, GF-IV and GF-V. Chin. Sci. Bull. 01: 011.

Chen YZ, Huo YS, Yu ZS and Zhang W (1986). The protective effect of Different parts of ginsenosides on myocardial necrosis. China J. Chin. Mat. Med. 6: 031.

Cui M, Song F, Zhou Y, Liu Z, et al. (2000). Rapid identification of saponins in plant extracts by electrospray ionization multi-stage tandem mass spectrometry and liquid chromatography/tandem mass spectrometry. Rapid Commun. Mass Spectrom. 14: 1280-1286. http://dx.doi.org/10.1002/1097-0231(20000730)14:14<1280::AID-RCM26>3.0.CO;2-C

Dey L, Zhang L and Yuan CS (2002). Anti-diabetic and anti-obese effects of ginseng berry extract: comparison between intraperitoneal and oral administrations. Am. J. Chin. Med. 30: 645-647. http://dx.doi.org/10.1142/S0192415X02000648

Fuzzati N (2004). Analysis methods of ginsenosides. J. Chromatogr. B Analyt. Technol. Biomed. Life Sci. B 812: 119-133. http:// dx.doi.org/10.1016/S1570-0232(04)00645-2

Guo JF, Zhong DF, Qiao SY and Zhao YM (2003). Analysis of ginsenosides by liquid chromatography-electrospray mass spectrometry. J. Chin. Mass Spectrom. Soc. 24: 477-481.

Han J, Ye M, Xu M, Sun J, et al. (2007). Characterization of flavonoids in the traditional Chinese herbal medicine-Huangqin by liquid chromatography coupled with electrospray ionization mass spectrometry. J. Chromatogr. B Analyt. Technol. Biomed. Life Sci. 848: 355-362. http://dx.doi.org/10.1016/j.jchromb.2006.10.061

Harkey MR, Henderson GL, Gershwin ME, Stern JS, et al. (2001). Variability in commercial ginseng products: an analysis of 25 preparations. Am. J. Clin. Nutr. 73: 1101-1106. 
Huang LL, Cheng WS, Chen YD and Hou F (2013). Research progress in ginseng fingerprint. Chin. Tradit. Herbal Drugs 44: 241-246.

Li L, Liu CM, Wu W, Yue H, et al. (2005). Analysis of saponins in extract of Panax Ginseng and Panax quinquefolius by liquid chromatography-electrospray ionization mass spectrometry. Chin. J. Anal. Chem. 33: 1087-1090.

Li X, Xiong Z, Ying X, Cui L, et al. (2006). A rapid ultra-performance liquid chromatography-electrospray ionization tandem mass spectrometric method for the qualitative and quantitative analysis of the constituents of the flower of Trollius ledibouri Reichb. Anal. Chim. Acta 580: 170-180. http://dx.doi.org/10.1016/j.aca.2006.07.069

Li XG and Teng FT (1978). The study of ginseng active ingredients (2) the comparison of different medicinal parts of Ginseng containing saponins and sapogenins. Chin. Trad. Patent Med. 4: 6-9.

Pharmacopoeia of the People's Republic of China (2010). 1st Part. China Medical Science Press, Beijing, 8-9.

Shi W, Wang YT, Quan XJ, Bai LF, et al. (2006). Determination of ginsenosides in the root of radix ginseng by high performance liquid chromatography/evaporative light scattering detection. Chin. J. Anal. Chem. 34: 243-246.

Xie JT, Zhou YP, Dey L, Attele AS, et al. (2002). Ginseng berry reduces blood glucose and body weight in db/db mice. Phytomedicine 9: 254-258. http://dx.doi.org/10.1078/0944-7113-00106

Xie P, Chen S, Liang YZ, Wang X, et al. (2006). Chromatographic fingerprint analysis--a rational approach for quality assessment of traditional Chinese herbal medicine. J. Chromatogr. A 1112: 171-180. http://dx.doi.org/10.1016/j.chroma.2005.12.091

Yahara S and Tanaka O (1979). Further study on dammarane-type saponins of roots, leaves, flower-buds, and fruits of Panax ginseng C.A. Meyer. Chem. Pharm. Bull. (Tokyo) 27: 88-92. http://dx.doi.org/10.1248/cpb.27.88

Yu M (2002). The study of the chemical constituents of ginseng fruit. Thesis from Liaoning Zhongyiyao Daxue.

Zhao S (1979). The study of ginseng fruit component. J. Tradit. Chin. Med. 12: 50-53.

Zheng Z, Song FD, Liu SY and Liu ZQ (2012). Fingerprint study on ginsenoside in ginseng and red ginseng. J. Chin. Mass Spectrom. Soc. 33: 327-334. 